\title{
Diseño y construcción de un instrumento prototipo para la medición de campo magnético homogéneo sinusoidal en baja frecuencia
}

\section{Design and construction of a prototype device for measuring sinusoidal homogeneous magnetic fields in low frequencies}

\section{Mario Alberto González Muñoz}

Tecnólogo en electricidad. Operador de Subestaciones de FYR Ingenieros Ltda. Bogotá, Colombia.kllride@hotmail.com

Clasificación del artículo: Investigación (Recreaciones)

Fecha de recepción: 23 de mayo de 2011

Fecha de aceptación: 29 de agosto de 2011

Palabras clave: Bobina Helmholtz, campo magnético homogéneo, filtro LC, solenoide.

Key words: Helmholtz coil, homogeneous magnetic field, LC filter, solenoid.

\section{RESUMEN}

En este artículo se describe el diseño y construcción de un equipo prototipo para la medición de campo magnético homogéneo sinusoidal con frecuencia de trabajo de $60 \mathrm{~Hz}$, utilizando como principal herramienta un sensor de efecto Hall.

El medidor cuenta con tres etapas de construcción, patronamiento del sensor de efecto Hall, programación del microcontrolador y visualización en pantalla. El patronamiento cuenta con dos etapas, filtrado de la señal de alimentación a la bobina de Helmholtz y calibración del sensor de efecto Hall. La parte final es la simulación del campo magnético en COMSOL como verificación del funcionamiento, tanto del elemento generador como del equipo medidor.

\section{ABSTRACT}

This paper describes the design and construction of a prototype equipment for measuring homogeneous magnetic field with working frequency sinusoidal $60 \mathrm{~Hz}$, using as the main tool hall effect sensor.

The meter has three stages of construction, patterns hall effect sensor, microcontroller programming and screen display. The employer has two stages, gating power to the Helmholtz coil and calibration of the Hall Effect sensor. The final part is the simulation of the magnetic field performance verification COMSOL as both a generator and the measuring equipment. 


\section{INTRODUCCIÓN}

Una problemática que existe en la actualidad es la falta de calidad y seguridad integral en el sector eléctrico y al mismo tiempo el incumplimiento del Retie en la instalación y diseño de estructuras y equipos eléctricos.

El grupo de investigación CEM (Compatibilidad electromagnética) de la Universidad Distrital Francisco José de Caldas, Facultad Tecnológica está dedicado al estudio de interferencias electromagnéticas, campos eléctricos, campos magnéticos y su objetivo es el de encontrar formas de atenuar o eliminar estas interferencias, al mismo tiempo que estudiarlas y comprender su existencia; asociado con estos temas se genera el proyecto diseño y construcción de un instrumento prototipo para la medición de campos magnéticos homogéneos sinusoidales en baja frecuencia, como necesidad para la medición de este fenómeno físico el cual está presente en todo momento.

El estudio realizado permite medir la incidencia de campo magnético que se genera sobre un equipo, y al mismo tiempo, comparar las lecturas con lo establecido en el reglamento nacional, dándole así una utilidad mayor y una utilización a futuro para que se pueda emplear en el análisis y la incidencia que tienen los campos magnéticos en los equipos electrónicos y en las instalaciones en general.

\section{FUNDAMENTOS TEÓRICOS}

\subsection{Campos magnéticos}

El fenómeno del magnetismo fue conocido por los griegos desde el año 800 a.C. Ellos descubrieron que ciertas piedras, ahora conocidas como magnetita $\left(\mathrm{Fe}_{3} \mathrm{O}_{4}\right)$, atraían piezas de hierro. En 1269 Pierre de Maricourt, mediante un imán na- tural esférico elaboró un mapa de las direcciones tomadas por una aguja al colocarla en diversos puntos de la superficie de la esfera. Encontró que las direcciones formaban líneas que rodeaban la esfera pasando a través de dos puntos diametralmente opuestos el uno del otro a los cuales llamo, los polos del imán.

El campo magnético se conoce como una región de espacio en la cual una carga eléctrica puntual de valor q, que se desplaza a una velocidad $\mathbf{V}$ y sufre los efectos de una fuerza que es perpendicular y proporcional, tanto a la velocidad v como al campo B. Así, dicha carga percibirá una fuerza descrita con la siguiente igualdad [1-3].

$$
F=q v \times \mathrm{B}
$$

\subsubsection{Campo magnético homogéneo}

Se dice que el campo magnético es homogéneo, cuando las partículas cargadas se mueven en una trayectoria circular, cuyo plano es perpendicular a B (Campo Magnético) y su intensidad depende del diseño y composición de la bobina que se utilice y de la fuerza magnética que se ejerce sobre la carga [3].

Esto indica que la fuerza magnética está en una dirección perpendicular tanto a la velocidad como al campo magnético que tiene un comportamiento homogéneo.

La magnitud de F se expresa de la siguiente forma:

$$
|F|=q|v||B| \sin \sin \theta
$$

Donde $\theta$ es el ángulo formado entre el campo magnético y la velocidad de la carga. Cuando la velocidad $\mathrm{v}$ es opuesta al campo magnético, $\mathrm{B}$, $\mathrm{F}=0$. Cuando el ángulo entre velocidad y campo 


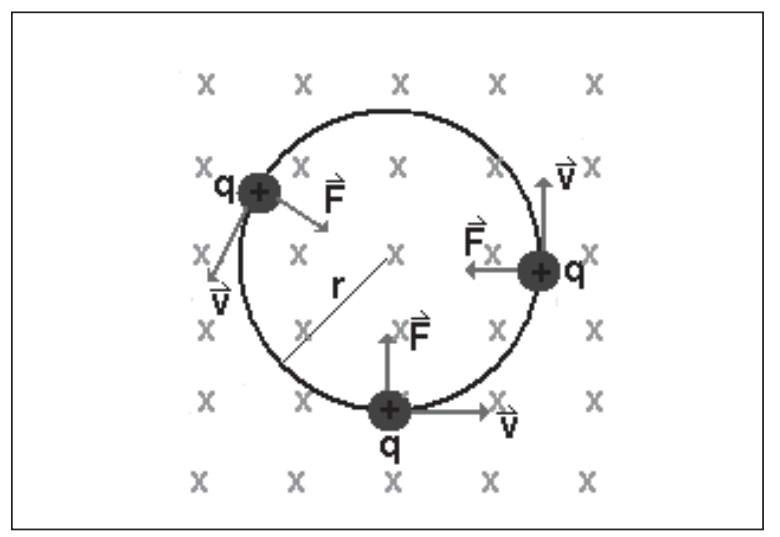

Fig. 1. Comportamiento de un campo magnético homogéneo.

Fuente: Serway Raymond, Electricidad y Electromagnetismo, Tercera edición, pág. 186.

es $0, F=q v B$, esto cuando la velocidad $\mathrm{v}$ es perpendicular al campo magnético, $\mathrm{B}$.

En la Fig. 1, se observa el comportamiento de una carga, q, en un campo magnético homogéneo. Se observa que la fuerza magnética se dirige al centro, para este caso de la bobina. Lo cual el campo magnético $\mathrm{B}$, entra a la bobina, como lo representan las cruces. Un campo homogéneo implica una intensidad de campo magnético permanente y constante, que no presente oscilaciones en los dos extremos de la bobina, en especial, el centro de la misma el campo sea constante.

\subsection{Ley de ampere}

La ley de Ampere es una descripción matemática del campo magnético, al mismo tiempo semejante y diferente al campo eléctrico, en el sentido que el campo magnético es una manifestación del campo eléctrico cuando intervienen movimientos relativos entre las fuentes. En el caso de configuraciones simples de corriente, la ley de Ampere describe cómo la fuente primordial de campo magnético son cargas eléctricas en movimiento [3].

La corriente en un alambre rectilíneo crea circunferencias de campo magnético constante alrede- dor de éste y como este campo es constante en cada circunferencia se calcula fácilmente la integral de línea a lo largo de cada circunferencia.

Esta integral es igual a la intensidad de corriente circulante en el alambre, multiplicada por una constante.

$$
\int B \cdot d r=4 \pi k_{m} I
$$

Ese resultado es el mismo para cualquier circunferencia y para cualquier trayectoria a lo largo del alambre. La integral de campo magnético en una trayectoria cerrada es igual a la constante de permeabilidad magnética por la intensidad de corriente que pasa por el alambre.

A esto se le conoce como ley de Ampere:

$$
\int B \cdot d r=\mu_{0} \cdot I
$$

\subsection{Ley de Biot-Savart}

Jean Batiste Biot y Feliz Savart, con sus resultados experimentales llegaron a una expresión de la que se obtiene el campo magnético en un punto dado del espacio en términos de la corriente que produce el campo [3].

La ley de Biot-Savart establece que si un alambre conduce una corriente constante I, el campo magnético $\mathrm{dB}$ en un punto $\mathrm{P}$ debido a un elemento $\mathrm{ds}$ tiene las siguientes propiedades.

- El vector $\mathrm{dB}$ es perpendicular tanto a ds (el cual tiene la dirección de la corriente) como al vector unitario ř dirigido desde el elemento hasta el punto $\mathrm{P}$.

- La magnitud de $\mathrm{dB}$ es inversamente proporcional a r2 donde $r$ es la distancia desde el elemento hasta el punto $\mathrm{P}$.

- La magnitud de $\mathrm{dB}$ es proporcional a la corriente y a la longitud ds del elemento. 
- La magnitud $\mathrm{dB}$ es proporcional al ángulo a sen $\theta$, donde $\theta$ es el ángulo entre el vector ds $\mathrm{y} \check{\mathrm{r}}$.

La ley de Biot-Savart puede ser resumida en:

$$
\mathrm{dB}=\left(\frac{\mu_{0}}{4 \pi}\right) *\left(\frac{\mathrm{d} \times R}{\mathrm{r}^{2}}\right)
$$

La ley de Biot-Savart proporciona el valor del campo magnético en un punto dado para un pequeño elemento del conductor. Para encontrar el campo magnético total $\mathrm{B}$ en algún punto debido a un conductor de tamaño finito, se deben sumar todas las contribuciones de todos los elementos de corriente que constituyen el conductor.

\subsection{Efecto Hall}

El efecto Hall es el fenómeno aprovechado en el presente proyecto, con el propósito de medir el campo magnético, gracias a una diferencia de potencial generada. Descubierto en 1879 por Edwin Hall, el fenómeno del efecto Hall muestra que cuando un conductor que es atravesado por una corriente se coloca en presencia de un campo magnético, se crea una diferencia de potencial perpendicular a la corriente y al campo magnético, debida a una desviación de los portadores de carga hacia uno de los lados del conductor, resultado de la fuerza experimentada por los portadores de carga.

El efecto Hall consiste en la aparición de un campo eléctrico en un conductor cuando es atravesado por un campo magnético. Cuando por un material conductor o semiconductor circula una corriente eléctrica, y estando este mismo material en el seno de un campo magnético, se comprueba que aparece una fuerza magnética en los portadores de carga que los reagrupa dentro del material, los portadores de carga se desvían y agrupan a un lado del material conductor o semiconductor,

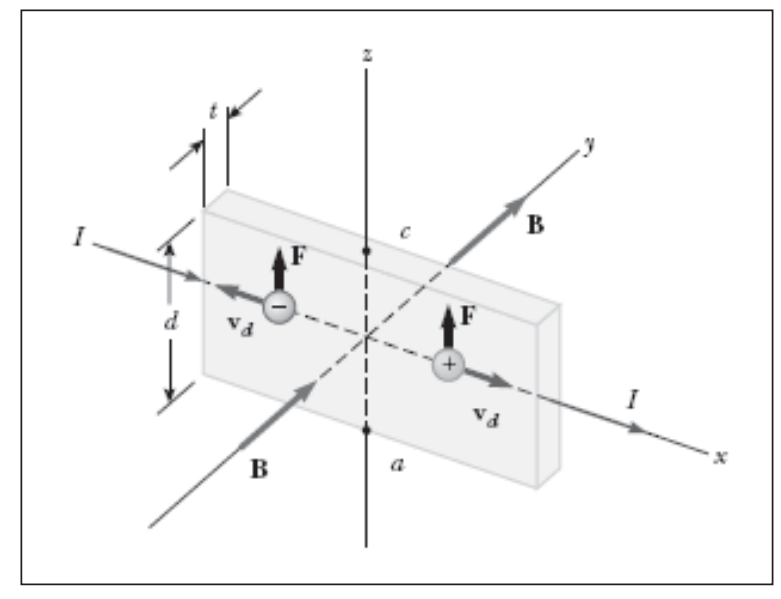

Fig. 2. Efecto Hall.

Fuente: Serway Raymon, Electricidad y Electromagnetismo, Tercera edición, pág. 194.

apareciendo así un campo eléctrico perpendicular al campo magnético [1 - 3].

Al hacer circular una corriente eléctrica por una placa de cualquier elemento e introducirla en un campo magnético uniforme y perpendicular a la placa aparecerá entonces una fuerza magnética sobre los portadores de carga, que tenderá a agruparlos a un lado de la placa, apareciendo de este modo una diferencia de potencial en los extremos de la placa, conocida como voltaje Hall y un campo eléctrico entre ambos lados de la barra. Dependiendo de si la lectura del voltímetro con el que se mida la diferencia de potencial es positiva o negativa y conociendo el sentido del campo eléctrico originado por la corriente eléctrica que circula por la placa y del campo magnético, podemos deducir si los portadores de carga de la placa de material desconocido son las cargas positivas o las negativas.

Una expresión para el voltaje Hall se deduce sabiendo que la fuerza magnética que actúa sobre los portadores de carga tiene una magnitud de $q v_{d} B$, al estar en equilibrio queda balanceada por la fuerza electrostática $q v_{d} E_{H}$, siendo $E_{H}$ el campo eléctrico debido a la separación de las cargas y mejor conocido como campo Hall. 


$$
\begin{gathered}
q v_{d} B=q E_{H} \\
E_{H=} v_{d} B
\end{gathered}
$$

Siendo $d$ el ancho del conductor, el voltaje Hall es igual a $E_{H d}$.

$$
V_{H=} E_{H} d=V_{d} B d
$$

Por lo tanto el voltaje Hall da un valor para la velocidad que deriva de los portadores de carga si se conoce $d$ y $E$.

El número de portadores de carga por unidad de volumen o (densidad de carga), $n$ se obtiene midiendo la corriente en la muestra y la velocidad que deriva se expresa como:

$$
V_{d}=\frac{1}{n q A}
$$

Siendo A el área transversal de la sección del conductor $A=t d$, donde $t$ es el espesor de la muestra utilizada para la recepción del campo.

$$
V_{d}=\frac{I B}{n q t}
$$

\section{METODOLOGÍA}

\subsection{Patronamiento del sensor de efecto Hall UGN3503 (allegro)}

Los sensores de efecto Hall son básicamente conductores en forma de placa por la cual circula una corriente y estos al ser expuestos a un campo magnético generan un voltaje, se escogió el UGN3503 allegro, por sus especificaciones en cuanto a extrema sensibilidad, baja salida de ruido, comportamiento estable a temperaturas superiores al ambiente y nivel de alimentación [4,5].

$\mathrm{Su}$ alimentación está desde $4.5 \mathrm{Vdc}$ a $6 \mathrm{Vdc}$, contiene una baja salida de ruido la cual permite que se atenúe usando un apantallamiento de ca- ble coaxial a la salida de voltaje. Su resolución depende de su alimentación dándose desde 0.75 hasta $1.75 \mathrm{mVac} /$ Gauss aunque en la práctica se encontró que alimentándolo al máximo voltaje de $8 \mathrm{Vdc}$ que soporta, proporciona una resolución máxima de $3 \mathrm{mV} /$ Gauss, cuenta con un ancho de banda de $10 \mathrm{~Hz}$ hasta $10 \mathrm{kHz}[6,7]$.

\subsection{Bobina de Helmholtz}

La bobina de Helmholtz consiste en dos solenoides de igual radio $\mathrm{R}$ y separadas por una distancia igual a su radio, si ambas espiras tienen un número de arrollamiento igual a $\mathrm{N}$ y por ambas espiras circula una corriente I (en el mismo sentido), se tiene que el campo magnético en el punto $\mathrm{P}$ de separación de los solenoides es constante dentro de un volumen de radio $\mathrm{R}^{3}[6,8]$. La expresión matemática que define la contribución de campo magnético en el punto $\mathrm{P}$ está dada por:

$$
B=2 *\left(\frac{\mu_{0} N I}{2 L}\left[\frac{z+L}{\sqrt{(z+L)^{2}+R^{2}}}-\frac{z}{\sqrt{z^{2}+R^{2}}}\right]\right)
$$

La bobina de Helmholtz utilizada en patronamiento del sensor cuenta con 351 vueltas de alambre esmaltado calibre 22, con un radio de $3.85 \mathrm{~cm}$ y una longitud de $25 \mathrm{~cm}$.

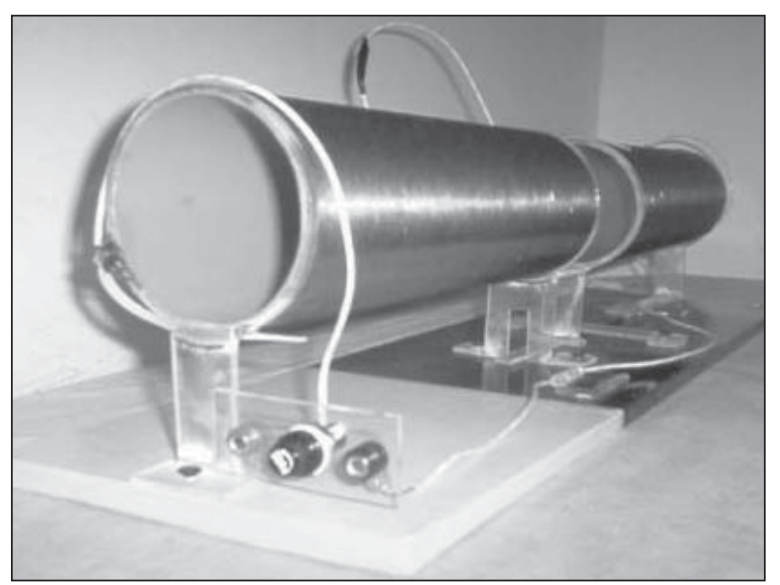

Fig. 3. Bobina de Helmholtz utilizada en el patronamiento del equipo. 


\subsection{Filtro LC}

Con el ánimo de evitar medidas erróneas y señales no deseadas en el patronamiento y calibración del sensor fue necesario implementar un filtro pasa baja el cual eliminará los componentes armónicos que se presentan en la alimentación de la bobina de Helmholtz, para esto se utilizó un circuito LC, el cual no tiene alta disipación de potencia en sus elementos, genera señales en una frecuencia particular y se puede almacenar energía eléctrica al vibrar su frecuencia resonante. Cuando la bobina del filtro y el condensador están conectados en paralelo, y Xc y Zl son iguales, la impedancia del filtro va a tender a infinito, y según su frecuencia de resonancia va a permitir el paso de solo ciertas frecuencias en la señal de corriente; su frecuencia de resonancia está dada por el despeje de w (frecuencia de corte) de la ecuación de impedancia del filtro [9].

Conociendo la impedancia de L y la reactancia de C se realiza el paralelo de los dos elementos.

$$
\text { Zfiltro }=\frac{\left(R_{L+j w L}\right)\left(-\frac{1}{j w C}\right)}{\left(R_{L+j w L}\right)+\left(-\frac{1}{j w C}\right)}
$$

$\mathrm{Al}$ obtener la parte real y la parte compleja de $\mathrm{Z}_{\text {filtro }}$ se iguala la parte compleja a 0 y se despeja $\mathrm{w}$.

$$
\begin{gathered}
R_{\text {filtro }=} \frac{\left(\frac{L}{C}\right)(R)-\left(\frac{R}{w C}\right)\left(w L-\frac{1}{w C}\right)}{(R)^{2}+\left(w L-\frac{1}{w C}\right)^{2}} \\
X_{\text {filtro }=R_{\text {fltro }}+j X_{\text {filtro }}}=\frac{\left(-\frac{R}{w C}\right)(R)-\left(\frac{L}{C}\right)\left(w L-\frac{1}{w C}\right)}{(R)^{2}+\left(w L-\frac{1}{w C}\right)^{2}}
\end{gathered}
$$

$$
\begin{gathered}
-\frac{R^{2}}{w C}-\frac{w L^{2}}{C}+\frac{L}{w C^{2}}=0 \\
w=\sqrt{\frac{1}{L C}-\left(\frac{R}{L}\right)^{2}}
\end{gathered}
$$

Los valores que se utilizaron en el filtro para el proyecto fueron obtenidos basándose en la ecuación (11), conociéndose el valor $w$ (frecuencia de corte) y manteniendo fijo el valor de la inductancia $L$, se despeja el valor de $C$, así se obtienen los valores de los elementos que componen el filtro, los parámetros de diseño del filtro son principalmente una reducción en el THD de la señal de alimentación, adicionalmente, los valores de frecuencia de las componentes armónicas se deben eliminar y solo permitir el paso de los valores de frecuencia iguales y menores a la del corte que se desea.

Con el analizador de energía Fluke disponible en el laboratorio de máquinas, se realizó la medida de voltaje sobre la bobina de Helmholtz, observando que en la señal de alimentación circulante por ésta, disminuía el THD y así teniendo en cuenta la ecuación (11) y con una frecuencia de corte de aproximadamente $250 \mathrm{~Hz}$ cercana al valor de frecuencia del tercer armónico, se obtuvieron los valores de capacitancia, manteniendo fijo el valor de inductancia.

Los valores necesarios para el filtro fueron de $\mathrm{L}=$ $10.5 \mathrm{H}$ con una resistencia de $124.5 \Omega$ y $\mathrm{C}=1.5$

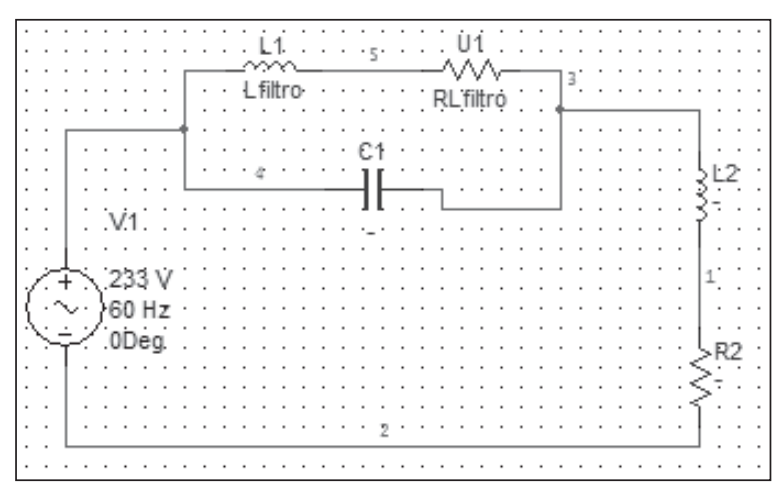

Fig. 4. Montaje experimental filtro LC. 


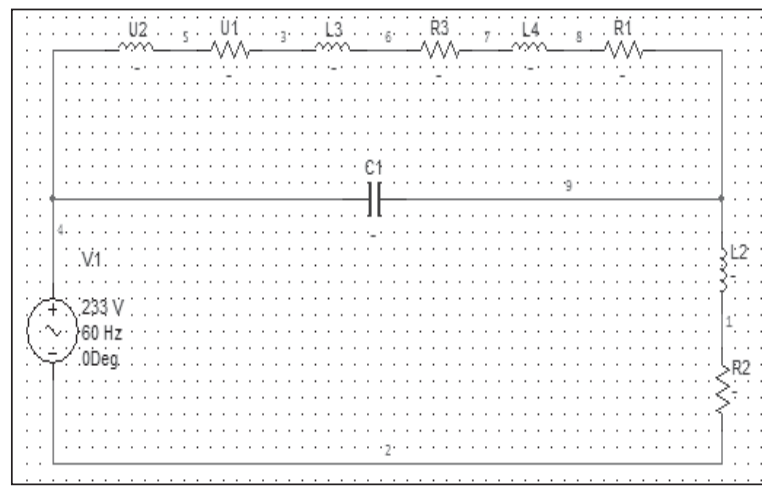

Fig. 5. Montaje filtro LC teniendo en cuenta Rbobinado.

$\mathrm{uF}$, a estos valores el filtro permite el paso de una señal de corriente con un THD de $2.3 \%$, el contenido armónico que tiene esta señal es de $\mathrm{H} 3 \% 2.0$, $\mathrm{H} 5 \% 0.9$ y $\mathrm{H} 7 \% 0.2$.

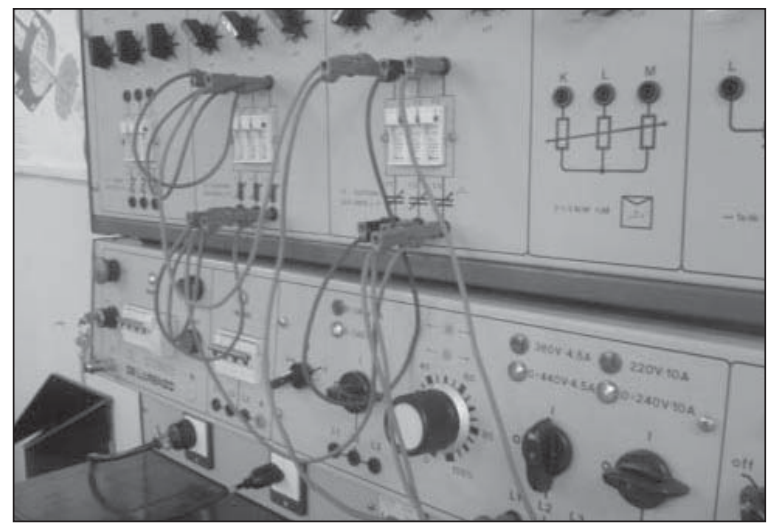

Fig. 6. Montaje real filtro LC del banco de pruebas.

\subsubsection{Simulación, sintonización e implemen- tación del filtro LC}

En el programa de circuitos Workbench se simuló la señal de alimentación del banco de pruebas como la suma de tres fuentes de tensión sinusoidal conectadas en serie alimentando una impedancia de $11.78+\mathrm{j} 45.2 \Omega$ la cual es equivalente a la impedancia de la bobina de Helmholtz, cada una de las fuentes tendrá la frecuencia asociada a la fundamental, del tercer y quinto armónico, así la conexión de las tres en serie simulará

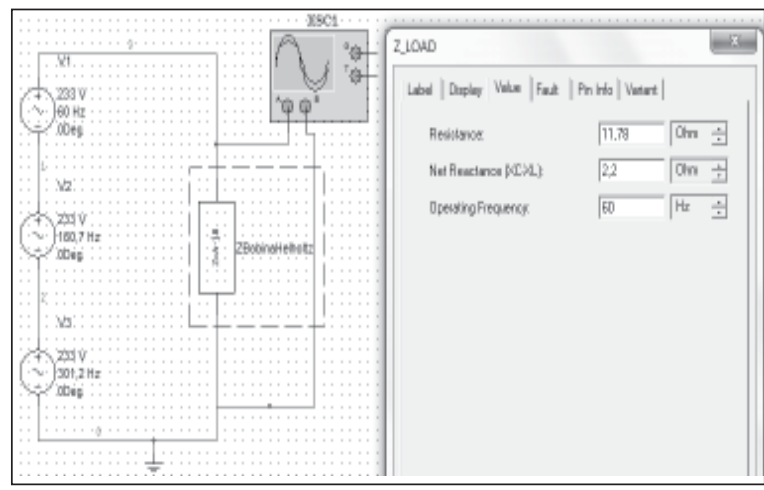

Fig. 7. Simulación, señal de alimentación del banco de pruebas a la bobina de Helmholtz por Workbench.

Tabla 1. Valores simulación, señal de alimentación del banco de pruebas.

\begin{tabular}{|l|c|c|}
\hline & $\begin{array}{c}\text { Magnitud de la } \\
\text { señal }\end{array}$ & Frecuencia \\
\hline Fuente 1. & $233[\mathrm{~V}]$ & $60 \mathrm{~Hz}$ \\
\hline Fuente 2. & $233[\mathrm{~V}]$ & $180.7 \mathrm{~Hz}$ \\
\hline Fuente 3. & $233[\mathrm{~V}]$ & $301.2 \mathrm{~Hz}$ \\
\hline Impedancia Helmholtz & $11.78+\mathrm{j} 45.2 \Omega$ & \\
\hline
\end{tabular}

aproximadamente la señal de voltaje del banco de pruebas [9].

Fig.9. Simulación filtro L, C por medio de Workbench.

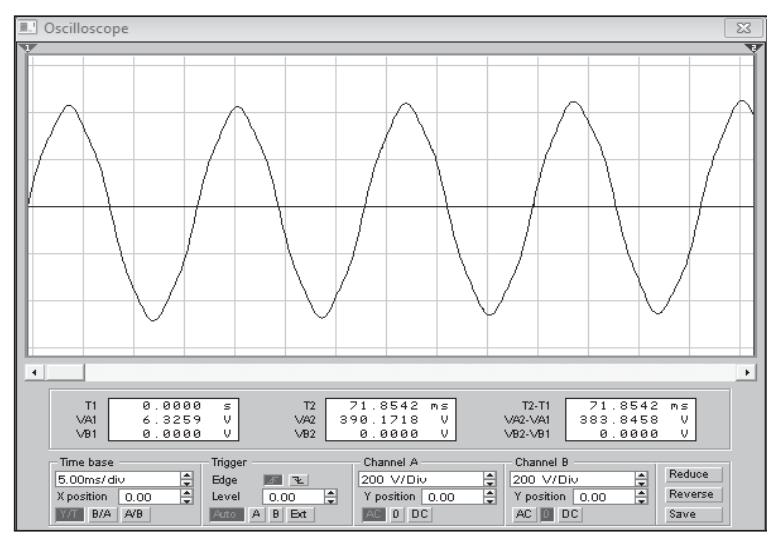

Fig. 8. Simulación señal de alimentación del banco de pruebas sin filtro. 


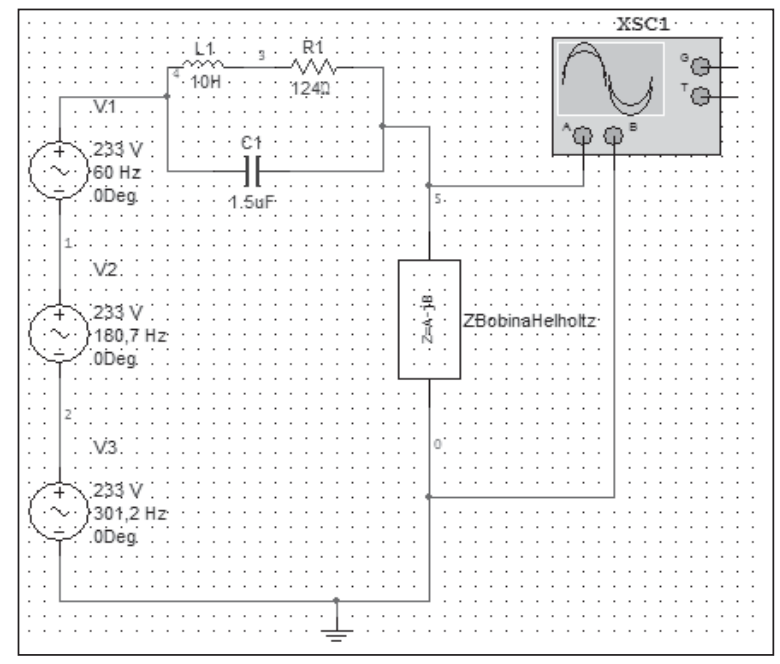

Fig. 9. Simulación filtro LC por medio de Workbench

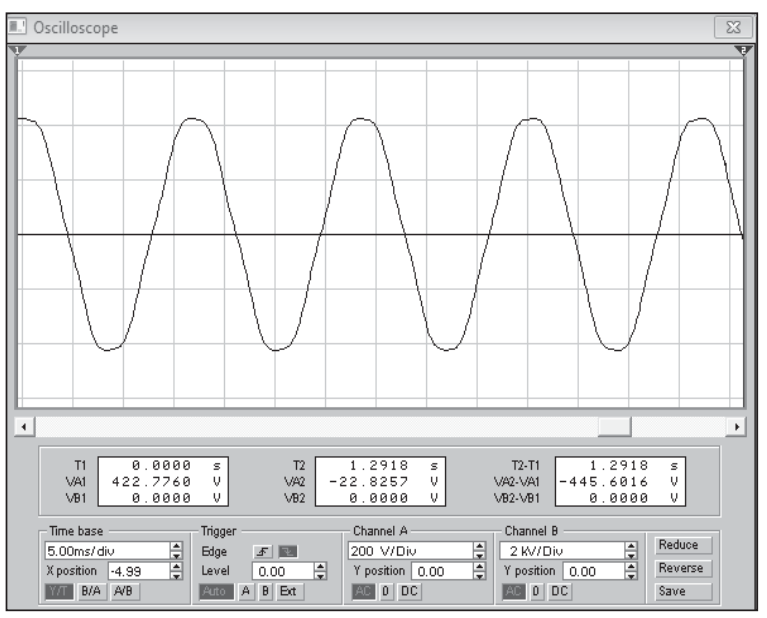

Fig. 10. Simulación señal de alimentación del banco de pruebas con filtro.

Tabla 2. Valores simulación filtro LC señal de alimentación del banco de pruebas.

\begin{tabular}{|l|l|l|}
\hline & \multicolumn{1}{|c|}{$\begin{array}{c}\text { Magnitud } \\
\text { de la señal }\end{array}$} & Frecuencia \\
\hline Fuente 1. & $233[\mathrm{~V}]$ & $60 \mathrm{~Hz}$ \\
\hline Fuente 2. & $233[\mathrm{~V}]$ & $180.7 \mathrm{~Hz}$ \\
\hline Fuente 3. & $233[\mathrm{~V}]$ & $301.2 \mathrm{~Hz}$ \\
\hline Impedancia Helmholtz & $11.78+\mathrm{j} 45.2 \Omega$ & \\
\hline Condensador filtro. & $1.5 \mathrm{uF}$ & \\
\hline Bobina filtro. & $10.5 \mathrm{H}$ & \\
\hline
\end{tabular}

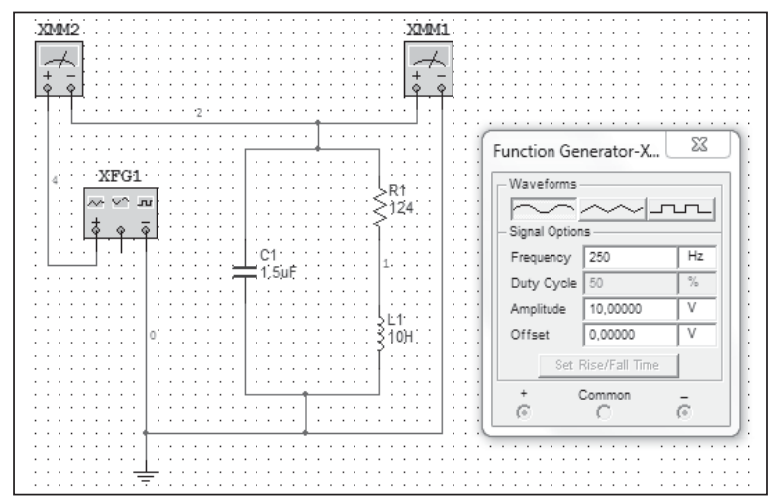

Fig.11. Montaje propuesto, barrido en frecuencia para filtro LC.

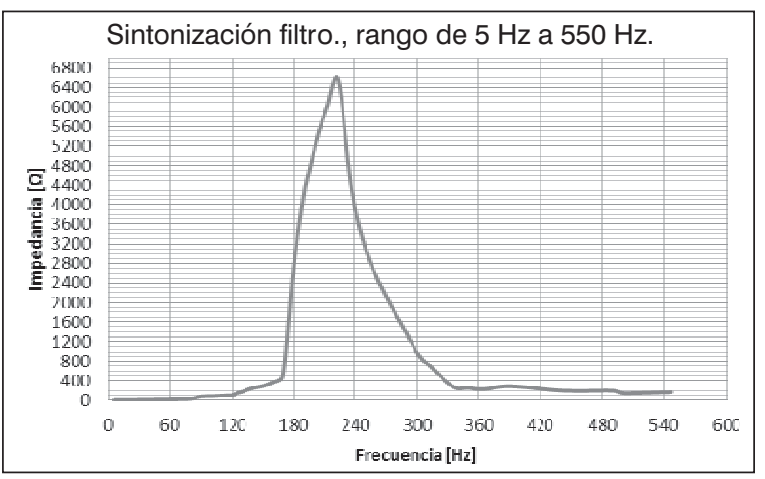

Fig. 12. Curva de sintonización rango de $5 \mathrm{~Hz}$ hasta $550 \mathrm{~Hz}$.

Para tener una mayor certeza de la fiabilidad del filtro fue necesario realizar una sintonización del mismo, con el fin de saber en qué valor de frecuencia se alcanzó el valor de impedancia más alto. Para esto se utilizó el generador de señales disponible en el laboratorio de máquinas de la Universidad Distrital Francisco José de Caldas con el cual se varió la frecuencia de una señal sinusoidal aplicada al filtro y se realizó medición de corriente y voltaje sobre una resistencia para poder saber el comportamiento de impedancia del filtro y así determinar en qué frecuencia está sintonizado.

En la Fig. 13. Se observa que el valor más alto de impedancia que toma el filtro es de $6550 \Omega$ a una frecuencia de $223 \mathrm{~Hz}$ aproximadamente, posterior a la sintonización del filtro LC se implementó el filtro en la alimentación de la bobina de Helmholtz 


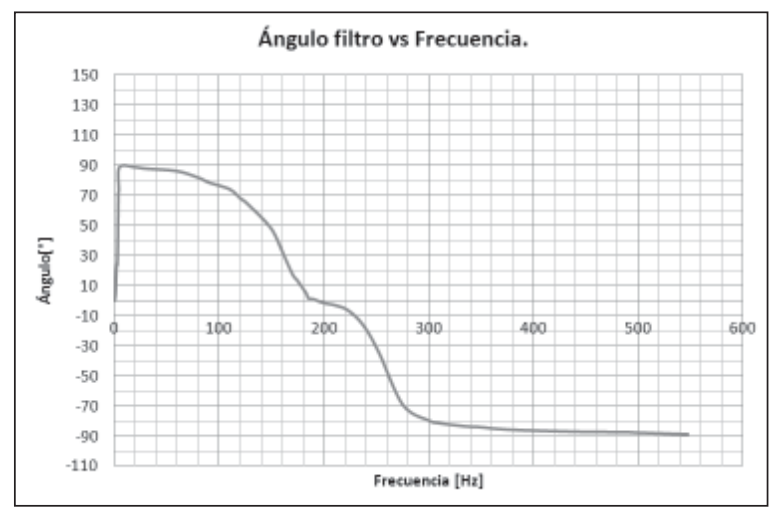

Fig. 13. Curva de ángulo de filtro vs frecuencia.

y se observó por medio del osciloscopio la señal y el contenido de armónicos resultante, siendo la una señal limpia con un THD de $2.3 \%$, tal como se esperaba en la simulación y la sintonización.

Se analiza por súper posición el circuito con el filtro, para comprobar que se tiene la intensidad de corriente necesaria en la señal de alimentación en la bobina de Helmholtz, tanto a $60 \mathrm{~Hz}, 180 \mathrm{HZ}$, y $301 \mathrm{~Hz}$, correspondientes al $1^{\circ}, 3^{\circ}$ y $5^{\circ}$ armónico respectivamente. Las corrientes obtenidas son:

Tabla 3. Resumen análisis de corriente en el filtro en frecuencias dif.

\begin{tabular}{|c|c|c|}
\hline$N^{\circ}$ de Armónico & Frecuencia & Intensidad de corriente \\
\hline $1^{\circ}$ & $60 \mathrm{~Hz}$ & $3,671 \cos (\mathrm{wt}-78,276)[\mathrm{A}]$ \\
\hline $3^{\circ}$ & $180 \mathrm{~Hz}$ & $0,085 \cos (\mathrm{wt}-2,767)[\mathrm{A}]$ \\
\hline $5^{\circ}$ & $301 \mathrm{~Hz}$ & $0,25 \cos (\mathrm{wt}+78,3)[\mathrm{A}]$ \\
\hline
\end{tabular}

Con los datos anteriores, se observó que la magnitud de la corriente que circula por el circuito cuando el filtro ataca el tercer armónico, se reduce su porcentaje en la señal, esto es debido a que el filtro se comporta como una impedancia muy grande y no permite el paso de esta frecuencia, lo mismo se observa en el análisis del quinto armónico, su porcentaje también se reduce, contrario a lo que ocurre con $60 \mathrm{~Hz}$ la frecuencia fundamental de la señal la cual es la que tiene el mayor porcentaje en la señal de alimentación a la bobina de Helmholtz.

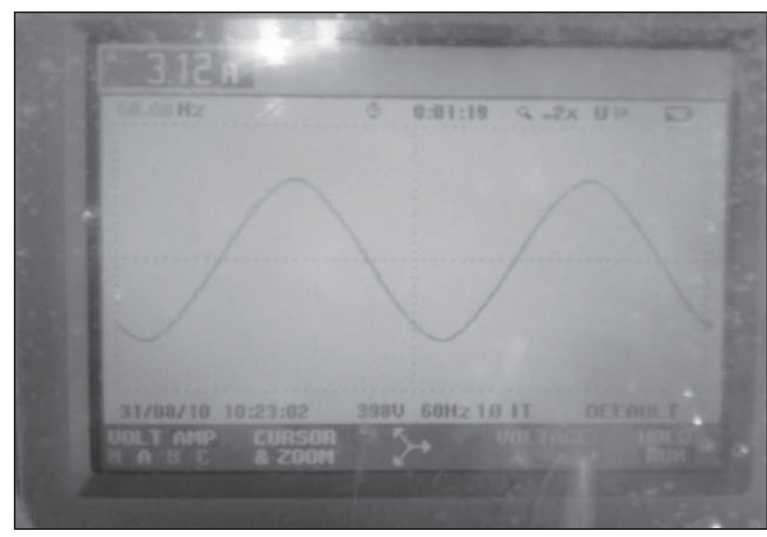

Fig.14. Señal de alimentación filtrada.

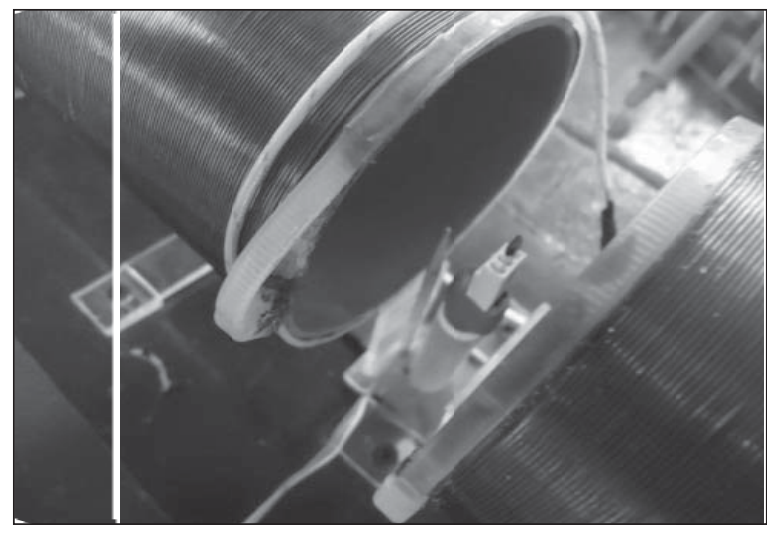

Fig.15. Patronamiento del sensor de efecto Hall UGN3503.

\subsection{Calibración sensor de efecto Hall UGN3503}

El sensor fue alimentado a $5 \mathrm{Vdc}$ que tiene una sensibilidad de $1.3 \mathrm{mV} / \mathrm{G}$, y expuesto a variaciones de campo magnético en un rango de corriente de $0[\mathrm{~A}] \mathrm{a} \pm 3.5[\mathrm{~A}]$ en corriente alterna, la temperatura máxima a la que fue expuesto fue de $35^{\circ} \mathrm{C}$ siendo ésta la de calentamiento de la bobina [7]. La temperatura de la bobina no afecta las mediciones realizadas al sensor de efecto Hall ya que el fabricante indica que la salida de voltaje empieza a aumentar a medida que la temperatura supera $\operatorname{los} 25^{\circ} \mathrm{C}$, cuando el sensor es expuesto a más de 500 Gauss, lo que equivale a 50 mTeslas, siendo 10 mTeslas el máximo valor de medición del equipo. 
El Patronamiento del sensor se realizó ubicándolo en la posición indicada dentro de la bobina de Helmholtz para poder realizar la medición del campo magnético homogéneo y se varió la corriente de alimentación de la bobina desde un valor de 0 [A] hasta $4[\mathrm{~A}]$ en corriente alterna y con un multímetro (true rms) se midieron las variaciones de voltaje a la salida del sensor.

Se deben realizar los cálculos del campo magnético teórico y graficarlos contra el voltaje Hall logrado en laboratorio para así obtener la ecuación de la recta, la cual proporciona la constante de calibración.

De la Fig.16 se adquiere la ecuación de la recta $\mathrm{Y}=1.4312 * \mathrm{x}+0.000009$, se obtiene la constante

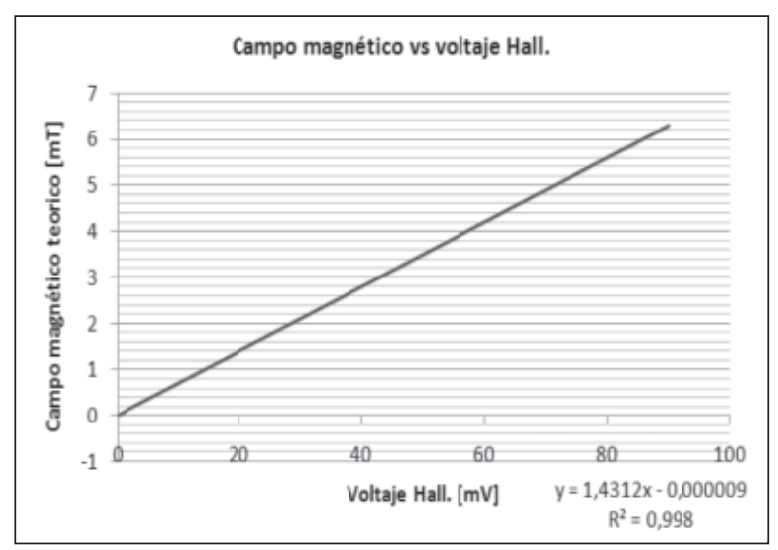

Fig. 16. Campo magnético teórico vs voltaje Hall.

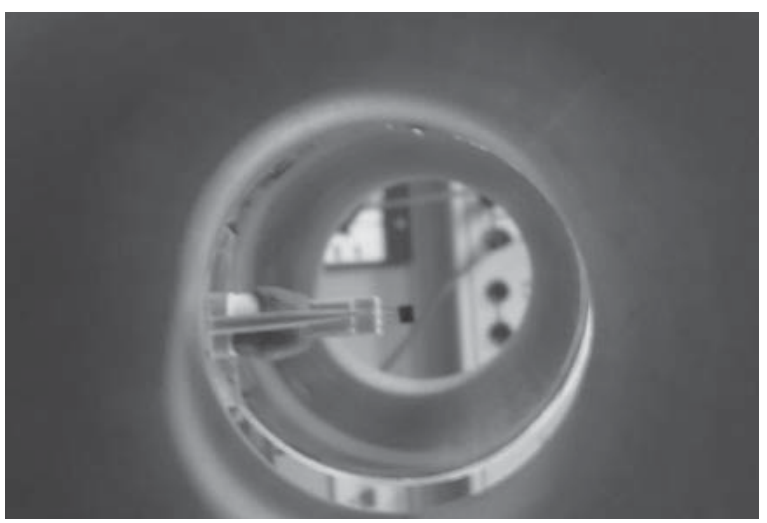

Fig.17. Calibración del sensor de efecto Hall UGN3503 de calibración siendo (1.4312) la constante y la suma de (0.000009) el Offset de la señal, así se consiguen los valores del campo magnético en la práctica.

\subsection{Etapa de programación}

El sensor de campo magnético allegro 3503 proporciona los datos de medición de campo magnético en voltios debido al principio de efecto Hall, la resolución de este se encuentra en milivoltios por Gauss y por tanto es necesario realizar una conversión de milivoltios por Gauss a milivoltios por Tesla, que en este caso se convierte a milivoltios por miliTeslas, ya que concuerda que 1 Gauss equivale 0.1 miliTeslas y así poder transferir estos datos a la pantalla LCD y visualizar la medición del campo magnético, para este proceso se necesita un elemento procesador el cual realice la conversión sin tener pérdida de datos en el proceso.

\subsubsection{Microcontrolador}

Para la programación se utilizó un dispositivo de programación, un microcontrolador, el cual es un circuito integrado de alta escala de integración que incorpora la mayor parte de los elementos que configuran un controlador.

El microcontrolador utilizado es un PIC 16F877A, es programado bajo leguaje C. y cuenta con un conversor análogo digital el cual es de gran utilidad, ya que la señal de salida del sensor es alterna [10].

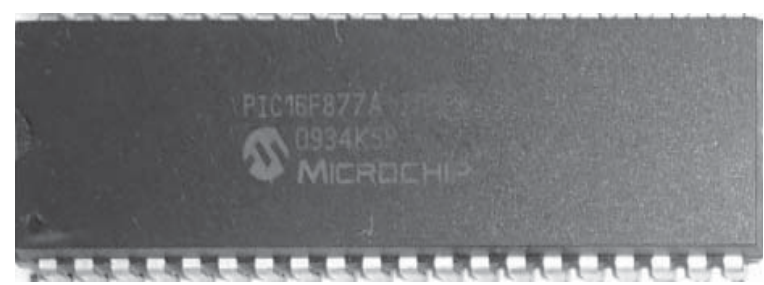

Fig. 18. Microcontrolador PIC 16F877A. 


\section{re-creaciones}

Tabla 4. Características Microcontrolador PIC 16F877A

\begin{tabular}{|l|c|}
\hline \multicolumn{2}{|l|}{ CARACTERísTICAS PIC 16F877A } \\
\hline Frecuencia de operación & DC - 20 MHz \\
\hline \multirow{2}{*}{ Resets y retrasos } & (PWRT, OST) \\
\cline { 2 - 2 } $\begin{array}{l}\text { Memoria de programación flash(14-bit } \\
\text { words) }\end{array}$ & $8 \mathrm{KOR}$ \\
\hline Memoria de datos (bytes) & 368 \\
\hline Memoria de datos EEPROM (bytes) & 256 \\
\hline Interruptores & 15 \\
\hline I/0 Puertos & Puertos A,B,C,D,E \\
\hline Temporizadores & 3 \\
\hline Módulos PWM/Comparación/Captura & 2 \\
\hline Comunicaciones seriales & MSSP,USART \\
\hline Comunicaciones paralelas & PSP \\
\hline Módulo análogo-digital 10-bit & 8 input channels \\
\hline Comparadores análogos & 2 \\
\hline
\end{tabular}

Fuente: Datasheet Microcontrolador PIC 16F877A.

\section{- Implementación de la programación en el} equipo

Para la conversión de datos a la salida del sensor de efecto Hall UGN3503 que está en milivoltios a miliTeslas y poder visualizarlos en la pantalla LCD, se realizó primero un patronamiento al microcontrolador para saber en qué rango se encontraba la señal de salida del sensor dentro del convertidor análogo-digital del microcontrolador, observándola en la pantalla LCD.

Posteriormente, se realizó la programación de éste en lenguaje c, bajo una compilación de 100 datos, cumpliendo con la resolución de 0.1 mTeslas y abarcando desde $0 \mathrm{mT}$ hasta $10 \mathrm{mT}$, tomando como principio la ecuación obtenida de la Fig.16. Esta ecuación se programó como base en el microcontrolador y en el pin 2 (RAO/AND) de entrada de datos se incluyeron las variaciones de salida del sensor de efecto Hall.

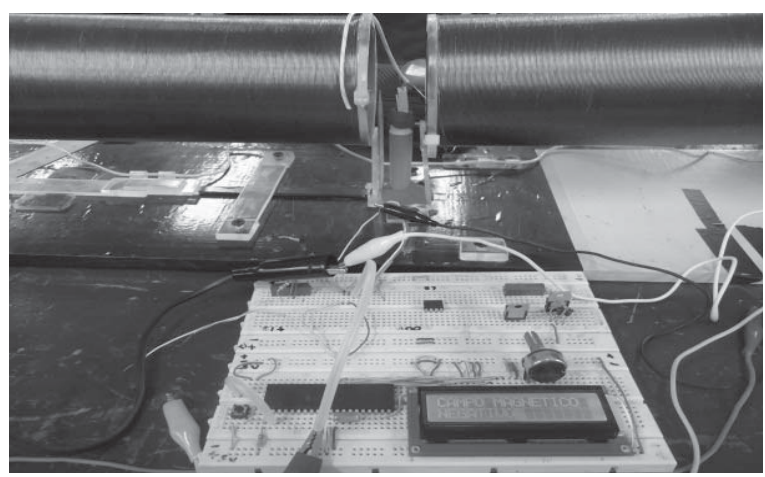

Fig. 19. Programación microcontrolador PIC 16F877A.

$\mathrm{Y}=1.4312 * \mathrm{x}+0.000009$, de esta ecuación se programó como constante multiplicadora el valor de (1.4312), x como entrada de datos del sensor y se le restó el valor de (0.000009), siendo este el offset que por defecto posee el sensor, programado esto se abrió la ventana del conversor análogodigital a 100 datos e incluyéndolos en el pin 2 con el valor de variación $\mathrm{x}$.

\subsection{Simulación etapa electrónica}

Por medio del simulador de circuitos electrónicos PROTEUS se corroboró que la programación aplicada al microcontrolador fue la correcta, y que al momento de realizar las medidas de campo magnético no se presentarían errores, se realizó una simulación del montaje de la tarjeta electrónica, la cual incluye la alimentación del equipo (incluye pantalla LCD, microcontrolador, Led de referencia y el sensor), y la programación del microcontrolador, dado que el programa PROTEUS no cuenta con la referencia del sensor UGN3503, se reemplazó en la simulación por un potenciómetro en el cual se varía la tensión eléctrica de entrada $[11,12]$.

Adicionalmente la simulación de la etapa electrónica cuenta con la visualización en pantalla de las mediciones de campo magnético indicando la equivalencia entre el voltaje de salida del sensor y el campo magnético calculado con la fórmula de la bobina de Helmholtz. 


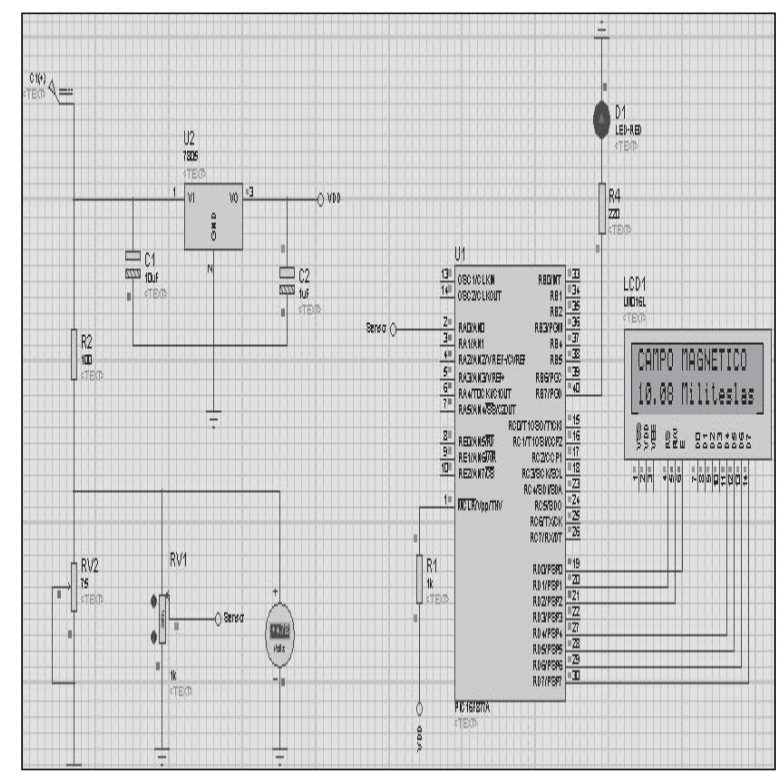

Fig. 20. Montaje simulación etapa electrónica.

\section{RESULTADOS}

\subsection{Presentación final y acabado del equipo}

El equipo en su acabado final se presenta en una caja de acrílico de $21.5 \mathrm{~cm}$ de largo, $9 \mathrm{~cm}$ de ancho, $4.3 \mathrm{~cm}$ de alto y con $3 \mathrm{~mm}$ de grueso de las placas, éstas lo protegen de golpes, derrames de líquidos, polvo y facilita su transporte para que sea más fácil realizar medidas a otras geometrías, cuenta con un interruptor de encendido/apagado y un Led de indicación que muestra el funcionamiento adecuado del microcontrolador cuando está encendido, la pantalla LCD es de $7 \mathrm{~cm} * 2.2 \mathrm{~cm}$.

La conexión y el transporte de datos del sensor al equipo se hace a través de un prototipo de sonda que consta de 3 cables utp donde van: la alimentación, punto de referencia o tierra y salida del sensor, dentro de un recubrimiento de cable coaxial que tiene apantallamiento en aluminio para evitar interferencias en la señal del sensor; la sonda se conecta a un acople Gp en el equipo el cual tiene las conexiones internas de alimentación, punto de referencia y salida de datos al microcontrolador.

\subsubsection{Jaula de Faraday}

Durante las pruebas de patronamiento se observó que a medida que el campo magnético aumentaba, éste afectaba el funcionamiento del microcontrolador, por tanto para proteger los componentes elec-

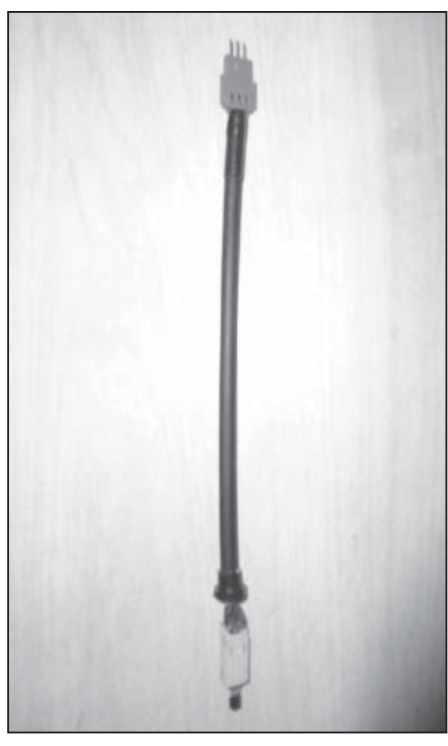

Fig. 21. Sonda Hall.

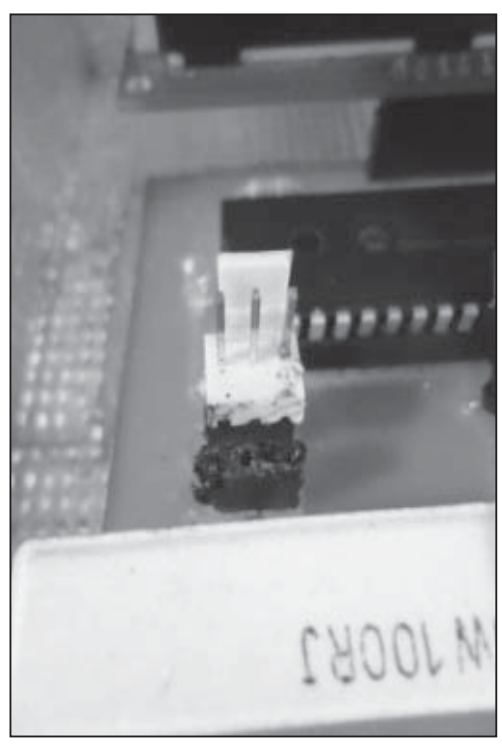

Fig. 22. Entrada de datos al equipo por acople Gp.

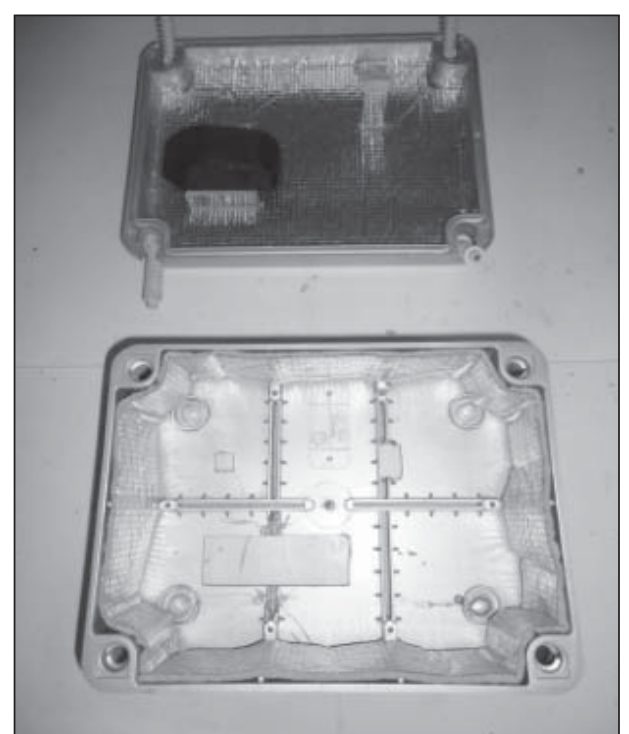

Fig. 23. Caja de protección con jaula de Faraday. 


\section{re-creaciones}

trónicos en el equipo se implementó una jaula de Faraday en aluminio pegada a la caja de acrílico; el funcionamiento de la jaula de Faraday se basa en las propiedades de un conductor en equilibrio electrostático. Cuando la caja metálica se coloca en presencia de un campo eléctrico externo, las cargas positivas se quedan en las posiciones de la red; los electrones, sin embargo, que en un metal son libres, se mueven en sentido contrario al campo eléctrico y, aunque la carga total del conductor es cero, uno de los lados de la caja (en el que se acumulan los electrones) se queda con un exceso de carga negativa, mientras que el otro lado se queda sin electrones (carga positiva) [2,3]

La jaula de Faraday utilizada para el prototipo es una lámina de espuma con un grosor de $5 \mathrm{~mm}$. para protección mecánica forrada por los dos lados con láminas de aluminio la cual cumple la función de jaula de Faraday.

\subsubsection{Alimentación del equipo}

El consumo del equipo es de $12 \mathrm{Vdc}$ a $175 \mathrm{~mA}$, y está alimentado a $18 \mathrm{Vdc}$ para mayor rendimiento por dos baterías recargables de $9 \mathrm{Vdc}$ de alto rendimiento (170 mAh), la distribución del voltaje se hace por medio de un regulador (7805) que proporciona $5 \mathrm{Vdc}$ al microcontrolador y a la panta1la LCD, la alimentación del sensor de efecto Hall UGN3503 se hace con un divisor de voltaje que consta de una resistencia de potencia y un trimer.

\subsubsection{Modo de uso y precauciones de operación}

Debido a la configuración geométrica del sensor de efecto Hall UGN3503 es preciso que la recepción del campo magnético que éste tenga sea perpendicular a la cara receptora, ya que no percibe campo magnético de la otra cara, además, el microcontrolador está programado para que cuando el campo esté entrando en esta cara se muestre en pantalla una anunciación de NE-

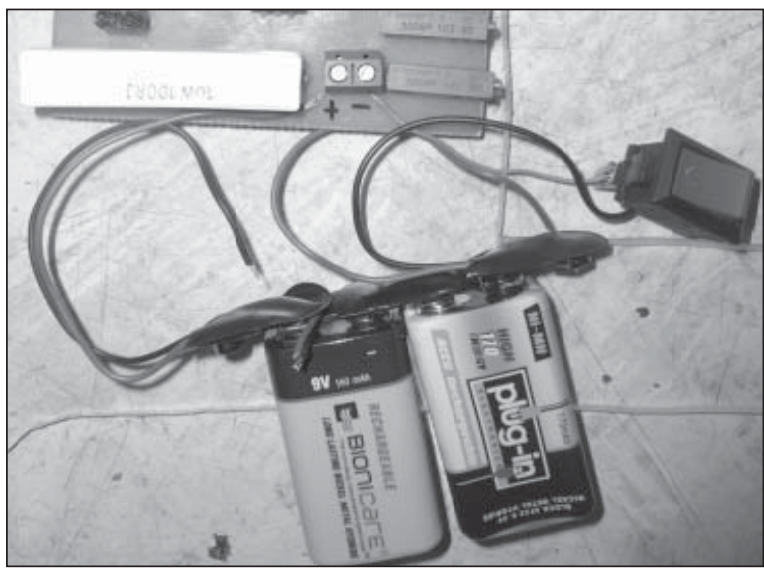

Fig. 24. Alimentación del circuito electrónico.

GATIVO, la cual indica que el campo va en la otra dirección.

La conexión Gp del equipo debe mantenerse en lo posible libre de polvo para evitar problemas en la salida del sensor al equipo, al igual que se recomienda evitar golpes al equipo y el contacto con líquidos y polvo excesivo, ya que esto podría dañar el microcontrolador y la pantalla LCD.

Las baterías tienen un tiempo de utilidad de 8 horas, después de esto las medidas y la pantalla van a presentar fallas, para esto se recomienda retirarlas y recargarlas en un tiempo de 12 horas, al pasar esto también es recomendable ajustar la alimentación del sensor con el trimer.

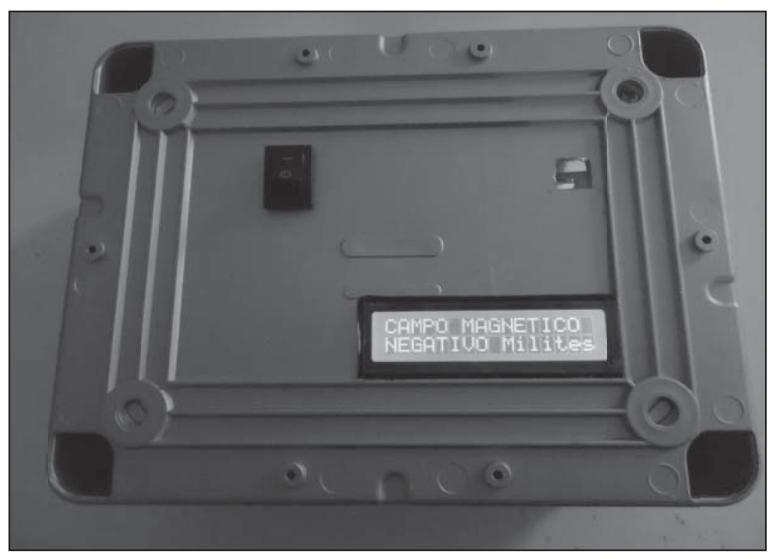

Fig. 25. Presentación final del equipo. 


\subsection{Simulación de etapa física por medio de COMSOL}

El programa de simulación COMSOL Multiphysics (COMSOL Multifisicas) que se conocía hace algunos años como FEMLAB (Laboratorio de fuerza electromagnética) es un software de análisis y respuesta por elementos finitos para varias aplicaciones físicas y de ingeniería, especialmente, fenómenos acoplados o de respuesta multifisica los cuales pueden ir de la simulación de un simple imán a la transferencia de calor que existe en un volcán o el movimiento de las capas tectónicas [13].

En el módulo en el que se realizan las simulaciones es el de AC/DC Module, que es el que cuenta con las aplicaciones de campos magnéticos, este módulo simula componentes y dispositivos eléctricos que dependen de fenómenos electrostáticos, magnetostáticos y aplicaciones electromagnéticas cuasiestáticas, particularmente acopladas a otras físicas. Consta de interfaces específicas para aplicaciones de maquinaria rotatoria e importación de listas de circuitos SPICE.

La simulación se realiza al elemento generador de campo magnético (la bobina de Helmholtz) la cual se construye en el programa como dos cilindros de cobre, respetando su geometría y haciendo circular por ellas una densidad de corriente equivalente a la corriente que circula por ellas en la práctica, la cual equivale como valor máximo a:

$$
J=14.18 * 10^{6}\left(\frac{A}{m^{2}}\right)
$$

Teniendo éste una equivalencia de 4 [A] y también con un valor intermedio de 2 [A] en el cual teóricamente el campo magnético es homogéneo en el punto de medición.

\subsection{Análisis de resultados}

A continuación se muestran las figuras obtenidas en las pruebas finales, después de haber pro-

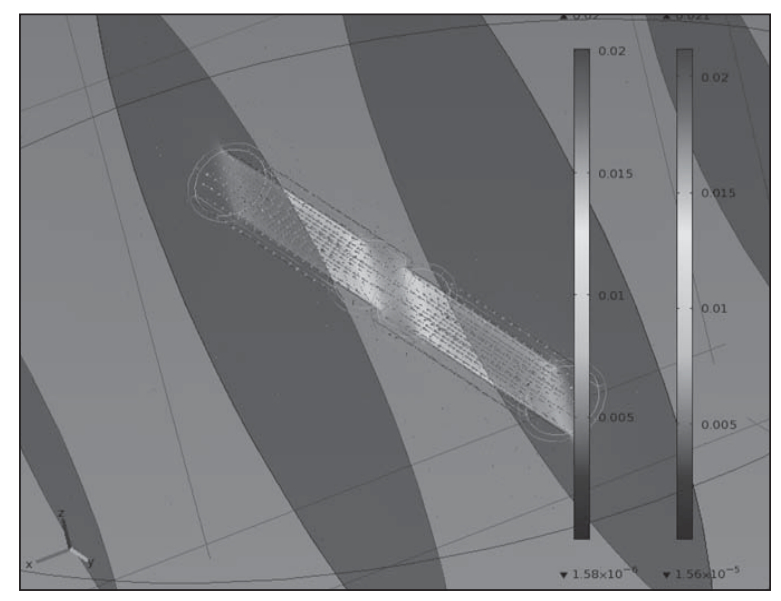

Fig. 26. Gráfica de densidad de campo magnético con vectores.

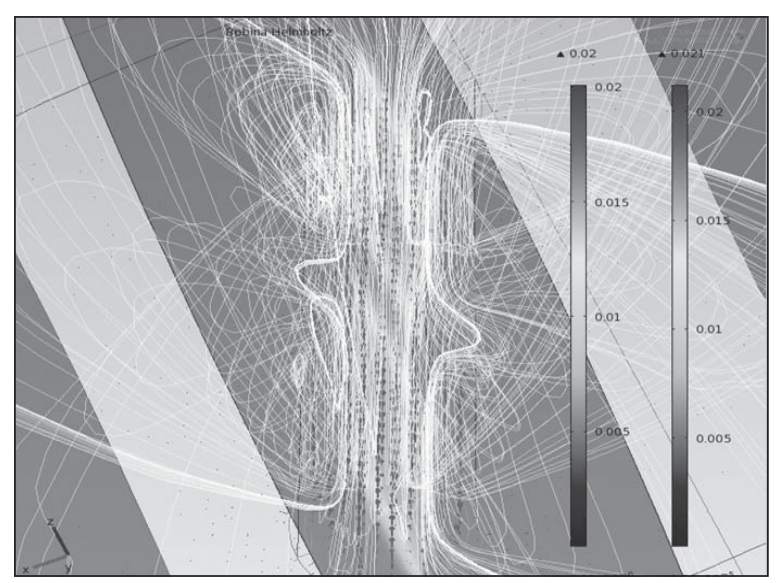

Fig. 27. Gráfica de densidad de campo magnético con vectores de campo y líneas de flujo magnético.

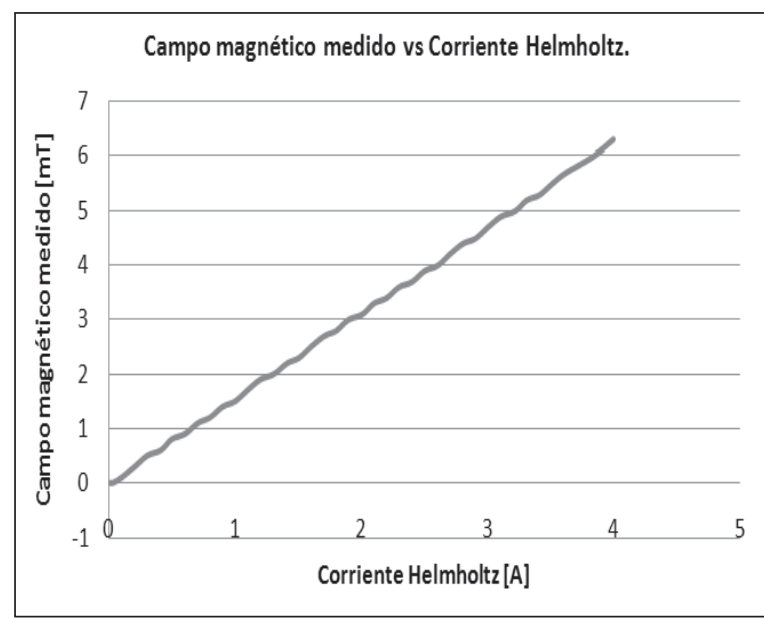

Fig. 28. Campo magnético teórico vs voltaje Hall. 


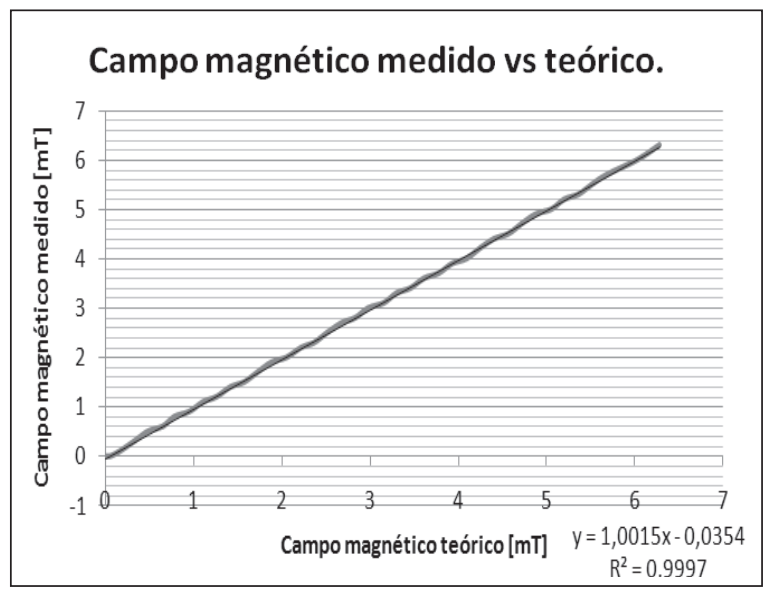

Fig. 29. Campo magnético teórico vs voltaje Hall.

gramado en el microcontrolador la ecuación: $y=$ $1.4312 * x-0.000009$ y realizando un último barrido de datos, para así observar una comparación de los datos obtenidos contra los datos esperados.

Estos resultados de medición y cálculo teórico concuerdan con la magnitud de campo magnético presentada de color azul claro en la simulación realizada en COMSOL.

Tabla 5. Costos generados en la construcción de la bobina de Helmholtz.

\begin{tabular}{|l|c|c|c|}
\hline Descripción & Cantidad & $\begin{array}{c}\text { Mano } \\
\text { de obra }\end{array}$ & $\begin{array}{c}\text { Costo } \\
\mathbf{( \$ )}\end{array}$ \\
\hline $\begin{array}{l}1 \text { tubo de plástico pvc, } \\
(3 ")\end{array}$ & 1 metro & 5000 \\
\hline $\begin{array}{l}\text { Alambre esmaltado ca- } \\
\text { libre 22 }\end{array}$ & 1.5 kilos & & 45000 \\
\hline Base de madera & $\left(64^{*} 18 \mathrm{~cm}\right)$ & & 3000 \\
\hline $\begin{array}{l}\text { Soportes y bases en } \\
\text { acrílico }\end{array}$ & 6 & 7000 & 5000 \\
\hline Bomeras (+-) & 2 & 1 & 1000 \\
\hline $\begin{array}{l}\text { Fusible de 4 [A] y por- } \\
\text { tafusible }\end{array}$ & $10 \mathrm{~mL}$ & & 15000 \\
\hline Esmalte dieléctrico & & 120000 & \\
\hline $\begin{array}{l}\text { Bobinado y ensamble } \\
\text { de la bobina de Hel- } \\
\text { mholtz }\end{array}$ & & & $\mathbf{2 0 2 3 0 0}$ \\
\hline Total & & & 1300 \\
\hline
\end{tabular}

Tabla 6. Costos generados en la construcción del equipo.

\begin{tabular}{|c|c|c|c|}
\hline Descripción & Cantidad & $\begin{array}{c}\text { Mano } \\
\text { de obra }\end{array}$ & $\begin{array}{l}\text { Costo } \\
(\$)\end{array}$ \\
\hline $\begin{array}{l}\text { Sensor de pruebas } \\
\text { Hall UGN3503 }\end{array}$ & 1 & & 13000 \\
\hline Sensores de prueba & 4 & & 50000 \\
\hline $\begin{array}{l}\text { Pantalla LCD de } 2 \text { * } 16 \\
\text { Caracteres }\end{array}$ & 1 & & 15000 \\
\hline $\begin{array}{l}\text { Microcontrolador PIC } \\
\text { 16F877A }\end{array}$ & 1 & & 11000 \\
\hline Portamicrocontrolador & 1 & & 2000 \\
\hline $\begin{array}{l}\text { Baquela en fibra de } \\
\text { vidrio }\end{array}$ & $6 * 8 \mathrm{~cm}$ & & 5000 \\
\hline $\begin{array}{l}\text { Resistores de } 10 \mathrm{Wy} \\
\text { de } 1 / 4 \mathrm{~W}\end{array}$ & 4 & & 1500 \\
\hline Trimers de $1 \mathrm{k} \Omega$ & 2 & & 2000 \\
\hline Regulador 7805 & 1 & & 1100 \\
\hline Acoples Gp & 5 & & 1500 \\
\hline Regleta de conexión & 1 & & 2000 \\
\hline $\begin{array}{l}\text { Conector } \\
\text { de alimentación }\end{array}$ & 1 & & 1000 \\
\hline Cristal de 2000 & 1 & & 2000 \\
\hline Led azul & 1 & & 500 \\
\hline Interruptor & 1 & & 300 \\
\hline $\begin{array}{l}\text { Caja de protección } \\
\text { con jaula de Faraday }\end{array}$ & 1 & & 10000 \\
\hline $\begin{array}{l}\text { Baterías de } 9 \mathrm{~V} \text {, con } \\
\text { cargador (1) }\end{array}$ & 2 & & 42000 \\
\hline Cachuchas de $9 \mathrm{~V}$ & 3 & & 1000 \\
\hline Sonda de datos & 1 & 5000 & 3000 \\
\hline $\begin{array}{l}\text { Programación } \\
\text { del microcontrolador }\end{array}$ & & 300000 & \\
\hline $\begin{array}{l}\text { Montaje en Baquela } \\
\text { y quemado del círculo }\end{array}$ & & 250000 & \\
\hline Total & & & 718900 \\
\hline
\end{tabular}

Los costos asociados al tiempo de realización del proyecto se hacen bajo el salario mensual de medio tiempo, del trabajo actual del autor del proyecto Mario Alberto González Muñoz. 
Tabla 7. Costos asociados al tiempo de realización del proyecto.

\begin{tabular}{|l|l|l|}
\hline Descripción & Tiempo & Costo. (\$) \\
\hline $\begin{array}{l}\text { Investigación y diseño del pa- } \\
\text { tronamiento }\end{array}$ & 4 meses & 2600000 \\
\hline Diseño del filtro pasa baja, L.C. & 1 mes & 650000 \\
\hline $\begin{array}{l}\text { Construcción de la bobina de } \\
\text { Helmholtz e implementación } \\
\text { del filtro }\end{array}$ & 1 mes & 650000 \\
\hline $\begin{array}{l}\text { Calibración del sensor de afec- } \\
\text { to Hall UGN3503 }\end{array}$ & 2 meses & 1300000 \\
\hline $\begin{array}{l}\text { Calibración del microcontrola- } \\
\text { dor }\end{array}$ & 2 meses & 1300000 \\
\hline $\begin{array}{l}\text { Programación del microcontro- } \\
\text { lador }\end{array}$ & 1 mes & 650000 \\
\hline $\begin{array}{l}\text { Construcción del equipo me- } \\
\text { didor }\end{array}$ & 1.5 meses & 975000 \\
\hline $\begin{array}{l}\text { Realización del documento fi- } \\
\text { nal }\end{array}$ & 1 mes & 650000 \\
\hline Total & 13.5 meses & 8775000 \\
\hline
\end{tabular}

Teniendo un costo total en el que se incluyen todos los componentes, investigaciones de realización y patronamiento del proyecto de $\$ 9.695 .300$.

\section{CONCLUSIONES}

Como cumplimiento del proyecto, se diseñó y construyó un instrumento prototipo medidor de campo magnético homogéneo, el cual tiene un valor mínimo de medición de $0 \mathrm{mT}$ y valor máximo de $10 \mathrm{mT}$, con una resolución de $0.1 \mathrm{mT}$ usando como transductor el mejor sensor de efecto Hall encontrado en el mercado, que muestra las lecturas en una pantalla adecuada para el uso de cualquier persona.

Para la etapa de patronamiento y calibración del sensor se investigaron diferentes geometrías de elementos generadores de campo magnético, los cuales cumplieran con los requerimientos mínimos de valor de campo magnético, llegando a la conclusión que el mejor elemento fue una bobina de Helmholtz, la cual consta de dos solenoides ubicados uno frente al otro para proporcionar un campo magnético homogéneo. Este elemento se implementó proporcionando los valores adecuados para el patronamiento del sensor utilizado en el proceso de medición del campo magnético.

Los cálculos y medidas realizadas en el proyecto fueron comprobadas exitosamente por medio del simulador de física COMSOL, el cual sirvió como herramienta de prueba para comprobar la homogeneidad del campo magnético en el punto de medición de las bobinas helmholtz y dar certeza de los valores que se midieron en la práctica.

La gran mayoría de sensores de efecto Hall utilizados durante la realización del proyecto presentaron mucha sensibilidad a interferencias de ruido eléctrico; esto se evidenció porque la señal de salida del sensor es un potencial eléctrico del orden de milivoltios, y puede ser afectada por interferencias. Por tanto se buscó en el mercado electrónico un sensor que cumpliera con los requerimientos en cuanto a rango, sensibilidad y precisión, así como con la condición de tener una baja sensibilidad a perturbaciones eléctricas externas, obteniendo el UGN3503, sensor con excelentes condiciones de operación tanto en temperatura, frecuencia y con una baja salida de tensión al ruido eléctrico.

Al filtrar la señal de alimentación se trató varias veces de implementar un filtro pasa baja de voltaje el cual eliminara los armónicos no deseados en la tensión de alimentación. El inconveniente que se presentó en un filtro de este tipo son los valores necesarios de capacitancia, resistencia y potencia disipada por estos para poder aplicarlo, por lo cual se utilizó un filtro pasa baja de corriente, que cumple la misma función de eliminar las componentes armónicas de la señal de alimentación, pero en la señal de corriente, que es la que genera las variaciones del campo magnético en la bobina de Helmholtz. Este filtro al ser implementado cumplió excelentemente el propósito de 


\section{re-creaciones}

poder alimentar la bobina de Helmholtz con una señal de corriente, la cual tuvo un THD de $2.3 \%$ para así poder realizar la medición de un campo magnético sin perturbaciones armónicas.

Al momento de realizar las pruebas de patronamiento al sensor, se alimentó la bobina de Helmholtz con corriente alterna y se midió la salida del sensor con un multímetro para registrar las variaciones del campo magnético en milivoltios, de igual forma, ocurrió con una alimentación en corriente directa, pero cuando la salida del sensor se acopló al microcontrolador al ser alimentado en corriente alterna la medida de campo magnético visualizada a través de la pantalla de LCD fue oscilatoria, aumentaba a medida que se incrementó la corriente, y descendía a 0 , luego volvió a aumentar, este comportamiento no se observó al energizar con corriente continua, puesto que la alimentación al elemento generador de campo magnético es alterna o en este caso sinusoidal; la señal de campo magnético [B] va a ser una señal que varía en el tiempo, comportamiento visualizado a través del microcontrolador.

Durante el proceso de investigación y construcción del equipo medidor se observaron varios medidores de campo magnético y eléctrico comunes en el mercado los cuales tenían costos mayores o iguales a \$US 2800 lo que equivale aproximadamente a $\$ 5^{\prime} 600.000$, realizando una comparación del costo que tiene un equipo manufacturado por una empresa reconocida, al construido en el proyecto se tendría un significativo ahorro de tres millones, lo cual indica que la consagración de este proyecto como empresa es mucho más accesible de lo que se piensa.

Al momento de enviar los datos de las variaciones proporcionados por el sensor de efecto Hall se pensó que debido a que la señal era muy pequeña y los valores se encontraban en milivoltios, era necesario realizar una amplificación, pero esto se solucionó enviando los datos a través de un conductor con apantallamiento en silicona y ajustando la resolución del microcontrolador para que éste capturara desde el mínimo valor de sensibilidad emitido por el sensor. Esto evitó la implementación de algún tipo de amplificador operacional o de instrumentación ya que estos pudieran amplificar señales de ruido y además aumentarían la carga de alimentación del circuito electrónico haciéndolo menos eficiente.

Al utilizar una bobina de Helmholtz, que en relación con una comercial tiene una mayor longitud en sus solenoides, se observó que el campo magnético en ésta permanece homogéneo en su punto de medición debido a que se elimina mejor el efecto de borde que se presenta en los solenoides, al igual que es una configuración poco utilizada en aplicaciones de laboratorio.

\section{AGRADECIMIENTOS}

A César Alexánder Chacón Cardona, físico y docente de la Universidad Francisco José de Caldas, Facultad Tecnológica, quien con su orientación, enseñanza, confianza me apoyó en todo momento, mis más sincero agradecimiento y respeto. 


\section{REFERENCIAS}

[1]. M. Alonso and E. Finn, Física Tomo 2. México: Addison Wesley Iberoamericana. 1999.

[2]. T. Croft, Tratado de electricidad práctica, $4^{\circ}$ Edición. Buenos Aires: Arbo. 1994.

[3]. A. Serway, Electricidad y Magnetismo, México: Mc Graw - Hill. (2001-2003).

[4]. T. Paul, "Magnetismo, luz y las físicas modernas elementales," $5^{\circ}$ Edición. En F. W. H., Física para los Cientificos y los ingenieros. 2004.

[5]. H. Stormer, "La Fracción cuantitativa del efecto Hall," Review of Modern Physics. 1999.

[6]. R. Wangness, Campos Electromagnéticos. México: Limusa. 1994.

[7]. Allegro Microsystems. Inc. Ratiometric, Linear Hall-Effect Sensors, 3503. Worcester, Massachusetts 1999.
[8]. Universidad Autónoma de Yucatán, Facultad de Ingeniería. Bobina de Helmholtz, Práctica 5. Yucatán. 2009.

[9]. D. Griffiths, La Introducción a la electrodinámica, $3^{\circ}$ Edición. Prentice Hall. 1998.

[10]. J. Angulo, I. Martínez, Microcontroladores Pic, Diseño Práctico de aplicaciones, $3^{\circ}$ Edición. Chile: Mac Graw Hill. 2005.

[11]. Boylestad and Nashelsky. Electrónica: Teoría de Circuitos, $6^{\circ}$ Edición. México: Pearson. 1997.

[12]. L. Agreement, COMSOL Multiphysics, Versión 4.0. Burlington, United States. 2010.

[13]. T. Paul, "Física para la ciencia y la tecnología,” En T. Paul, Física para la Ciencia y la tecnología. España: Reverté. 2005. 